\title{
Comparative study of four different types of intraperitoneal mesh prostheses in rats ${ }^{1}$
}

\author{
Rogério Aoki Fuziy' (D) , Ricardo Artigiani Neto" (D) , Elesiario Marques Caetano Junior" (D) , Ana Karina \\ Soares Alves"II (D) , Gaspar Jesus Lopes Filho'v (D) , Marcelo Moura Linhares ${ }^{\mathrm{V}}$ (D)
}

' Fellow Master degree, Postgraduate Program in Interdisciplinary Surgical Sciences, Division of Surgical Gastroenterology, Department of Surgery, Universidade Federal de São Paulo (UNIFESP), Brazil. Design of the study; acquisition, analysis and interpretation of data; manuscript preparation; critical revision; final approval.

" PhD, Division of Surgical Gastroenterology, Department of Surgery, UNIFESP, Sao Paulo-SP, Brazil. Design of the study; acquisition, analysis and interpretation of data; manuscript preparation; critical revision; final approval.

"'MD, Division of Surgical Gastroenterology, Department of Surgery, UNIFESP, Sao Paulo-SP, Brazil. Acquisition and interpretation of data, critical revision, final approval.

IVPhD, Chairman, Division of Surgical Gastroenterology, Department of Surgery, UNIFESP, Sao Paulo-SP, Brazil. Acquisition and interpretation of data, critical revision, final approval.

${ }^{\vee}$ PhD, Full Professor, Division of Surgical Gastroenterology, Department of Surgery, UNIFESP, Sao Paulo-SP, Brazil. Design of the study; acquisition, analysis and interpretation of data; critical revision; final approval.

\begin{abstract}
Purpose: To compare four types of mesh regarding visceral adhesions, inflammatory response and incorporation.

Methods: Sixty Wistar rats were divided into four groups, with different meshes implanted intraperitoneally: polytetrafluoroethylene (ePTFE group); polypropylene with polydioxanone and oxidized cellulose (PCD); polypropylene (PM) and polypropylene with silicone (PMS). The variables analyzed were: area covered by adhesions, incorporation of the mesh and inflammatory reaction (evaluated histologically and by COX2 immunochemistry).

Results: The PMS group had the lowest adhesion area (63.1\%) and grade 1 adhesions. The ePTFE and PM groups presented almost the total area of their surface covered by adherences $(99.8 \%$ and $97.7 \%$ respectively).The group ePTFE had the highest percentage of area without incorporation (42\%; $p<0.001)$ with no difference between the other meshes. The PMS group had the best incorporation rate. And the histological analysis revealed that the inflammation scores were significantly different.

Conclusions: The PM mesh had higher density of adherences, larger area of adherences, adherences to organs and percentage of incorporation. ePTFE had the higher area of adherences and lower incorporation. The PMS mesh performed best in the inflammation score, had a higher incorporation and lower area of adherences, and it was considered the best type of mesh.
\end{abstract}

Key words: Tissue Adhesions. Surgical Mesh. Cyclooxygenase 2. Rats. 


\section{- Introduction}

Incisional hernias are one of the most frequent postoperative complications ${ }^{1}$. Obesity, advanced age, diabetes, pulmonary diseases, malnutrition and multiple surgeries at the same site are predisposing factors. Several hernia correction methods have been proposed; yet none is considered the gold standard in the repair of incisional hernias, and the treatment of obese patients, with multiple hernia lesions or abdominal wall loss is even more complex ${ }^{2}$.

The repair of incisional hernias using simple closing techniques or the Mayo procedure is recommended to fix small defects with less than $5 \mathrm{~cm}$, but they may have a recurrence rate above $50 \%{ }^{3,4}$. For large incisional hernias, techniques using mesh present lower recurrence and hazard rates than techniques without mesh reinforcement, according to a recent metanalysis ${ }^{1}$. The use of mesh has indeed become standardized in incisional hernias, allowing the reduction of the recurrence rate to $5.2-24 \% 5,6$. Surgical treatment of large incisional hernias aims to repair the defect and to normalize the containment function of the abdominal wall, and synthetic prostheses reinforce the suture and replace missing tissue ${ }^{7}$. However, despite better results with synthetic prostheses than with simple closure, they can cause severe complications, depending on the positioning in which they are fixated on the abdominal wall ${ }^{8}$.

Three sites are suitable for the positioning of the mesh: on the aponeurosis, pre-peritoneally and intraperitoneally. Positioning on the aponeurosis is the most commonly used method, but it is associated with higher recurrence rate and higher incidence of postoperative complications, such as wound infection, hematoma and seroma formation ${ }^{8}$. In the pre-peritoneal position, the mesh is protected from the abdominal contents through the posterior fascia of the rectus abdominis muscle and peritoneum, or only the peritoneum, if it is positioned posteriorly to the fascia. This technique is described as leading to a lower rate of adhesion formation and fewer postoperative complications ${ }^{9}$.

Intraperitoneal position was used in the past, with interposition of the omentum on the abdominal viscera. Contact of the mesh with the intestines can occur when the peritoneum closure is not possible, and the surgeon tries a tension-free repair. The use of intraperitoneal mesh was associated with firm adhesions, intestinal lesions, migration and erosion of the mesh by the adjacent organs with formation of enterocutaneous fistula ${ }^{10,11}$, although the association of the fistula with the positioning of intraperitoneal polypropylene mesh has not been observed in all studies ${ }^{12}$.

To avoid this serious complication, the industry developed mesh screens coated with absorbable material, which act as a temporary barrier on the polypropylene framework and the abdominal viscera, such as the polypropylene mesh coated with hyaluronic acid, or nonabsorbable material, such as expanded polytetrafluoroethylene (ePTFE) and polypropylene coated with silicone ${ }^{13-16}$. The polypropylene material can also be embedded with anti-adherent solutions of hyaluronic acid or icodextrin or coated with methylcellulose, hyaluronic acid or titanium; the polyester mesh may be embedded with collagen ${ }^{17}$. The possibility of adding an absorbable non-adherent solution made with polyethylene glycol hydrogel has been investigated too ${ }^{18}$.

Each type of mesh is different in porosity and thickness, as well as density, which potentially influences the incorporation into the scar tissue and the foreign body reaction or biocompatibility ${ }^{19,20}$, as well as adhesions. However, although the studies have compared, usually two by two, different mesh materials for intra peritoneal repair, it is still necessary to evaluate the adherence of prosthetic mesh to abdominal viscera as well as its incorporation to the abdominal wall and a local inflammatory reaction. Therefore, the objective of the present study is to compare four different products regarding visceral adherences and tissue inflammatory reaction: a polypropylene mesh, a polypropylene mesh with encapsulated polydioxanone and coated with oxidized cellulose, a polypropylene mesh coated with silicone and a polytetrafluoroethylene expanded mesh. The hypothesis was that incorporation, adhesions and local inflammatory reaction would be different between the mesh materials.

\section{- Methods}

This research protocol was approved by the institutional ethics committee (protocol CEP 1292/11), as it fully adheres to all national and institutional guidelines for the care and use of the animals used in experimental researches, and also the current national laws.

\section{Study design, experimental animals and housing and husbandry}

This is an experimental surgical study with Wistar rats, comparing four different mesh support products for hernia repair. The experimental procedures were performed always in the same laboratory, a light and 
temperature-controlled room. All the animals used in this study had been bred in the same university bioterium, and were healthy before the experiment. All rats were male and weighted around 250 grams.

A convenience sample of 60 rats was used. Rats were identified with numbers and were maintained in groups of 5 animals per cage. They were observed during the study period for autophagia, mutilating behavior, infections and body movements. Rats were fed and hydrated ad libitum. The size of the cages was $30 \mathrm{~cm} \times 40 \mathrm{~cm} \times 20 \mathrm{~cm}$. After identification with numbers, the animals were randomly allocated in four groups of 15 animals using electronic random allocation sequence generation.

Four different compositions of mesh products were used in each group, as follows:

- ePTFE group: polytetrafluoroethylene expanded mesh (Gore-Tex Dual Mesh; Gore-tex United States);

- PCD group: polypropylene mesh encapsulated with polydioxanone and coated with oxidized cellulose (Proceed, Ethicon, United States);

- PM group: polypropylene mesh (Prolene, Ethicon, United States);

- PMS group: polypropylene mesh coated with silicone (Implants, Microval, France).

\section{Surgical procedures}

Rats were fasted for 8 hours prior to surgery. Anesthesia was made with ketamine hydrochloride in combination with xylazine ( $10 \%$ and $2 \%)$, by intraperitoneal injection, at a dose of $0.1 \mathrm{ml}$ of solution per $100 \mathrm{~g}$ of body weight. Trichotomy of the anterior abdominal wall, with electrical appliance, was followed by antisepsis with iodopovidine topical solution. Sterile techniques were used during all surgeries.

The surgical technique model used in this study was the one proposed by Alponatet al. ${ }^{13}$ and Hooker et al. ${ }^{14}$. A medial incision of $4 \mathrm{~cm}$ allowed the harvest of two skin patches that were separated from the abdominal wall, followed by the opening of the abdominal cavity through a longitudinal incision to the peritoneum, promoting a gap of $1.5 \times 2.5 \mathrm{~cm}$. All mesh products were cut into $3.5 \mathrm{~cm} \times 2.5 \mathrm{~cm}$ square patches (area pf $8.75 \mathrm{~cm}^{2}$ ). In all animals the meshes were positioned intra peritoneal and in contact with intra-abdominal viscera, with six separate sutures of polypropylene 5-0, three sutures on each side. Skin was sutured with separate points of Vicryl 4-0 (Figs. 1 and 2).

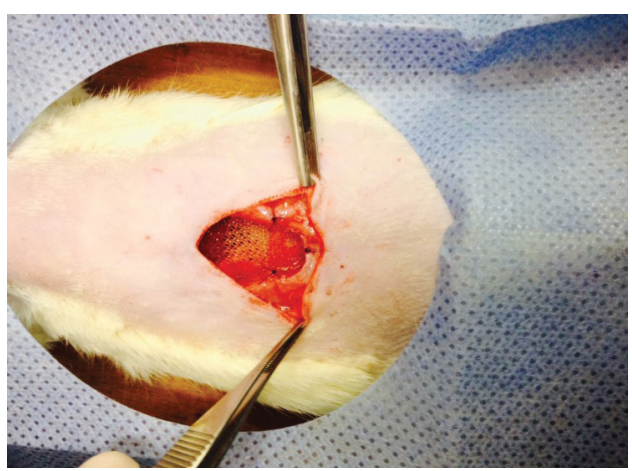

Figure 1 - Experimental incisional hernia repair: final aspect of the mesh fixation, in contact with viscera.

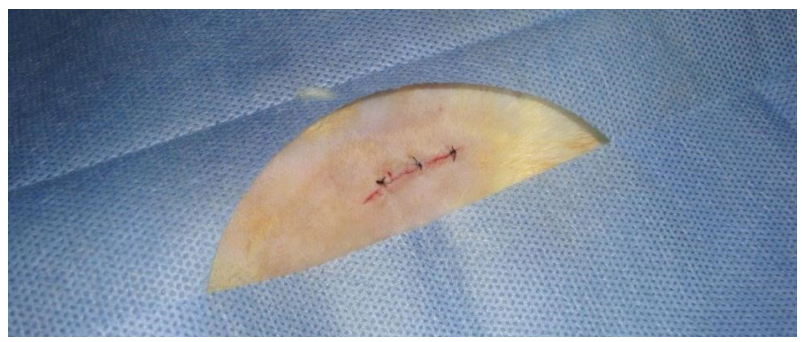

Figure 2 - Skin suture with Vicryl 4-0.

\section{Necropsy and macroscopic evaluation}

Euthanasia was performed in the seventh postoperative day, followed by necropsy for histological evaluation. Rats were euthanized using $0.5 \mathrm{ml}$ of ketamin and $0.5 \mathrm{ml}$ of xilazin intraperitoneally. After euthanasia, the abdominal cavity of each rat was open in a U-shaped incision (Fig. 3) that contained the lateral and inferior regions of the mesh. Cases of infection were identified when there was pus in the wound or on the mesh (Fig. 4). In the macroscopic evaluation, the pathologist evaluated if there were adherences of the mesh to abdominal organs and incorporation of the mesh to the abdominal wall (Fig. 5).

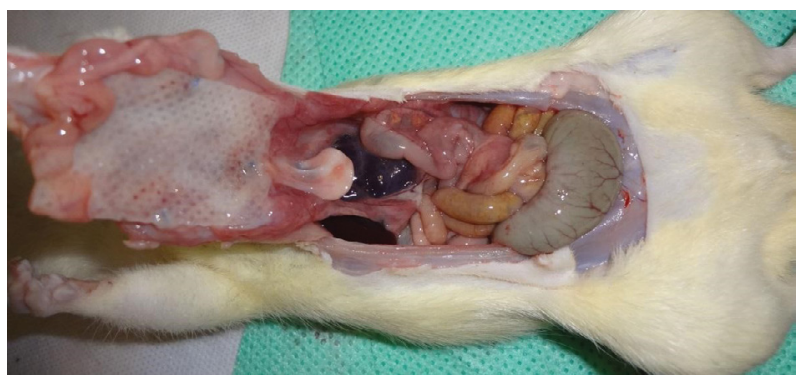

Figure 3 - U-shaped incision in the abdominal cavity of the Wistar rat, after euthanasia. 


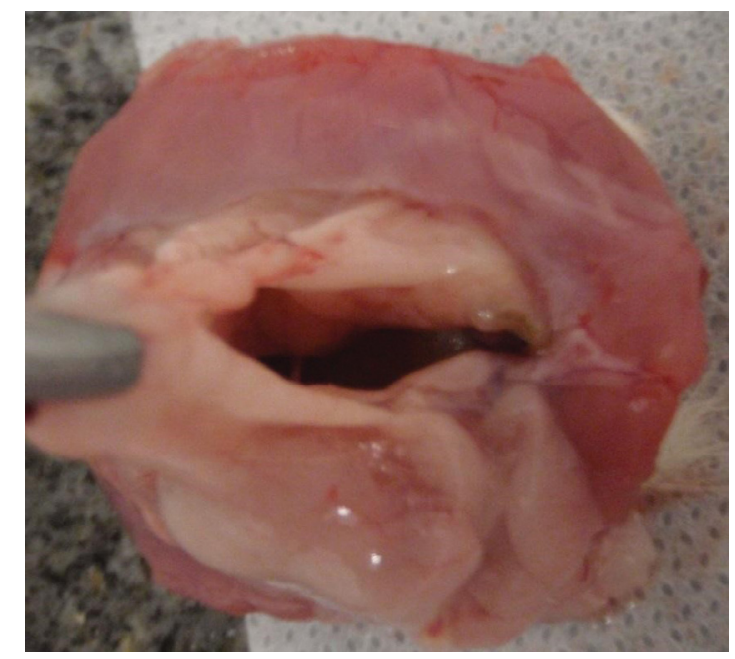

Figure 4 - Purulent secretion in the wound.

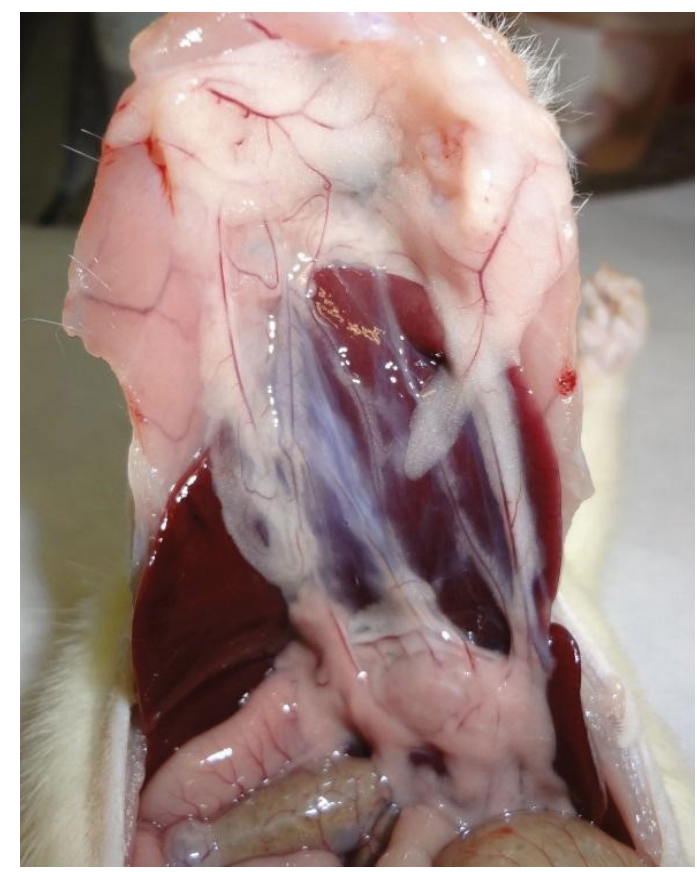

Figure $\mathbf{5}$ - Adherence of abdominal viscera to the mesh.

The mesh was cut together with tissues adhered to it and the roots of visceral organs close to it. For the evaluation of the density of adhesions, the mesh surface was divided in six larger fields, and each of these six sections subsequently subdivided in other six fields, and for each field, the percentage of the surface covered by adhesions was calculated. The adhesions density was then classified as follows ${ }^{15-17}$ :
Grade zero: no adherence;

Grade 1. Filmy adhesions, easy to separate by blunt dissection;

Grade 2, mild, but stronger adhesions, blunt dissection possible with partly sharp dissection;

Grade 3, moderate or strong adhesions, lysis possible, but with sharp dissection only;

Grade 4, very strong or severe adhesions, lysis is possible but with sharp dissection only; organ strongly attached can be damaged by dissection.

The incorporation area of the mesh to the omentum was measured by dividing the mesh in 10 sections and calculating the percentage of sections with incorporation (Fig. 6).

After these macroscopic evaluations, the material was fixed in buffered formalin (10\%) and sent for histological analysis.

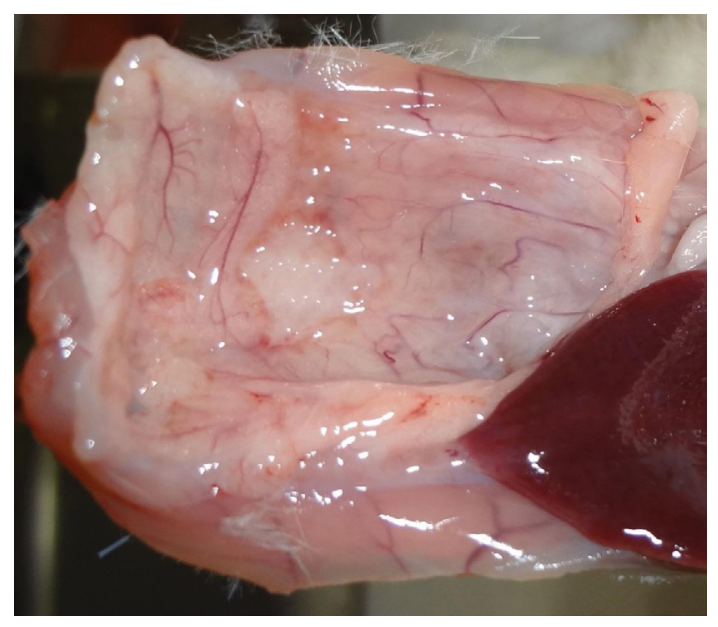

Figure 6 - Area to be considered in the incorporation evaluation.

\section{Histological and immunohistochemistry evaluation}

After fixation, the tissue samples were treated with xylene and paraffin embedding, and were subjected to microtomy and hematoxylin-eosin (HE; Fig. 7) and Masson trichrome staining. One single experienced pathologist from the university team made all histological and immunohistochemistry evaluations. This pathologist was blind to animal allocation. Initially, using optical microscopy with increase of $\times 200$, the field to be evaluated was chosen at the transition between the mesh and the host tissue. Histological evaluation was then performed with magnification of $\mathrm{x} 400$. 


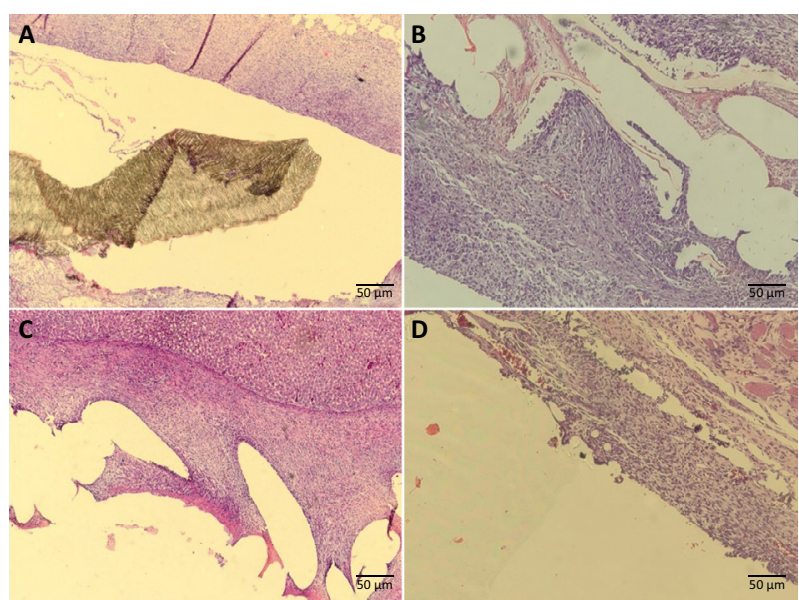

Figure 7 - Hematoxylin-eosin stained slides of the transition between the mesh and the host tissue: $\mathbf{A}$. ePTFE group: polytetrafluoroethylene expanded mesh; B. PCD group: polypropylene mesh encapsulated with polydioxanone and coated with oxidized cellulose; $\mathbf{C}$. PM group: polypropylene mesh, and D. PMS group: polypropylene mesh coated with silicone.

For the inflammatory reaction evaluation, we used the scale proposed by Harrell et al. ${ }^{18}$, with scores describing: A) the layers of cells in the periphery of the granulomas, with scores varying according to the number of layers; B) the inflammatory reaction in host tissue; C) the inflammatory response on the mesh surface; and D) the tissue maturation, with scores from 1 to 4 . Two complementary evaluation items of the inflammatory reaction were also added (also with score values from 1 to 4), a classification proposed by Pereira-Lucena et $a{ }^{19}{ }^{19}$. This intends to evaluate the presence of giant cells and inflammatory invasion of muscles adjacent to the mesh.

The paraffin embedded blocks were used to prepare slides for COX2 evaluation (polyclonal Spring antibodies) by immunohistochemistry (Fig. 8), with the following steps:

- Antibody identification;

- Antigenic recovery;

- Blocking of endogenous peroxidase;

- Incubation with the primary antibody;

- Incubation with the one-step polymer (LSAB kit, Dako, K0640);

- Revelation and counterstain (DAB kit, Dako);

- Dehydration and assembly.

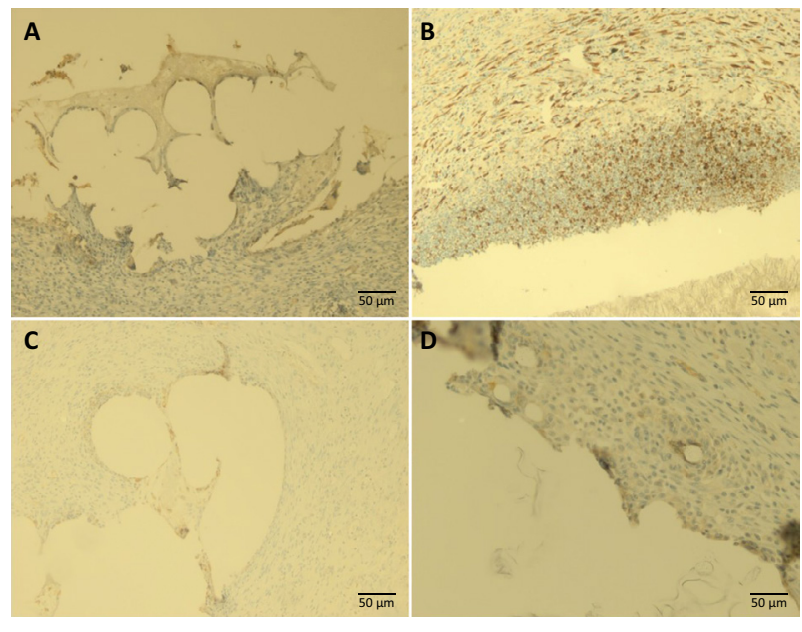

Figure 8 - COX-2 evaluation by immunohistochemistry: A. ePTFE group: polytetrafluoroethylene expanded mesh; B. PCD group: polypropylene mesh encapsulated with polydioxanone and coated with oxidized cellulose; C. PM group: polypropylene mesh, and D. PMS group: polypropylene mesh coated with silicone.

For objective data analysis, we used the Kim et al. ${ }^{20}$ scale, with expression scores based on the percentage of stained cells and the intensity of the reaction, as shown in Table 1. This analysis was performed both in the peritoneal and the non-peritoneal sides of the mesh, because the mesh products can have different surfaces in different sides.

Table 1 - Criteria for immunohistochemistry COX2 evaluation: amount of stained cells and intensity of reaction.

\begin{tabular}{lc}
\hline Percentage of stained cells & Points \\
\hline Up to $25 \%$ & 1 \\
$26 \%$ to $50 \%$ & 2 \\
$51 \%$ to $100 \%$ & 3 \\
Intensity of reaction & \\
Mild & 1 \\
Moderate & 2 \\
Strong & 3 \\
\hline
\end{tabular}

\section{Statistical analysis}

The Shapiro-Wilk test was used to test normality of quantitative variables. The chi-square and the Fisher's exact 
tests were used to verify associations between categorical variables and the four mesh types. For quantitative variables, the Kruskal-Wallis test was used, followed by paired Mann-Whitney test to better discriminate the statistical significance between two types of mesh.

The level of statistical significance was set at 0.05 . Analyses were performed using the SPSS statistical package (version 18.0).

\section{Results}

Two rats died during the experiments, one in the PMS group and the other in the PM group. Both deaths occurred in the immediate postoperative period and followed the increase of anesthetic doses for surgery. The study thus began with 14 animals in the PM and PMS groups and 15 in the PCD and ePTFE groups. However, it was not possible to perform immunohistochemistry analysis in all rats due to problems with the slides staining. COX-2 evaluation was performed in 14 animals in the PM and PCD groups, 13 animals in the PMS group and 12 animals in the ePTFE group.

The density of adhesions of the different mesh products is shown in Tables 2 and 3, while Table 4 shows the areas of lack of incorporation, with significant difference between groups. The results show that the incorporation area was the worst with the ePTFE group. Hematoma or seroma was present in all rats of the PTFE group, with a significant difference between groups (Table 5).

Table 2 - Adherence scores per type of mesh.

\begin{tabular}{|c|c|c|c|c|c|c|c|c|}
\hline $\begin{array}{l}\text { Adherence } \\
\text { score }\end{array}$ & ePTFE & PCD & PM & PMS & Total & Chi-squared & $\mathbf{p}$ & Fisher $\mathbf{p}$ \\
\hline Grade 1 & $0(0 \%)$ & $0(0 \%)$ & $0(0 \%)$ & $13(92.9 \%)$ & $13(22.4 \%)$ & \multirow{5}{*}{97.827} & \multirow{5}{*}{$<0.001$} & \multirow{5}{*}{$<0.001$} \\
\hline Grade 2 & $1386.7 \%)$ & $15(100 \%)$ & $1(7.1 \%)$ & $1(7.1 \%)$ & $30(51.7 \%)$ & & & \\
\hline Grade 3 & $2(13.3 \%)$ & $0(0 \%)$ & $4(28.6 \%)$ & $0(0 \%)$ & $6(10.3 \%)$ & & & \\
\hline Grade 4 & $0(0 \%)$ & $0(0 \%)$ & $9(64.3 \%)$ & $0(0 \%)$ & $9(15.50 \%)$ & & & \\
\hline Total & $15(100 \%)$ & $15(100 \%)$ & $14(100 \%)$ & $14(100 \%)$ & $58(100 \%)$ & & & \\
\hline
\end{tabular}

PTFE: polytetrafluoroethylene expanded; PCD: polypropylene encapsulated with polydioxanone and coated with oxidized cellulose; PM: polypropylene mesh; PMS: polypropylene mesh coated with silicone.

Table 3 - Estimated area of adherence per type of mesh.

\begin{tabular}{lcccc}
\hline $\begin{array}{l}\text { Estimated \% } \\
\text { ofadherencearea }\end{array}$ & PTFE & PCD & PM & PMS \\
\hline N & 15 & 15 & 14 & 14 \\
Mean & $99.87 \%$ & $83.20 \%$ & $97.71 \%$ & $63.14 \%$ \\
SD & $0.52 \%$ & $21.75 \%$ & $5.81 \%$ & $31.11 \%$ \\
Median & $100.00 \%$ & $92.00 \%$ & $100.00 \%$ & $70.00 \%$ \\
KW $\chi 2$ & & $\mathbf{2 5 . 8 1 6}$ & \\
-value & & $<\mathbf{0 . 0 0 1}$ & \\
Mann-Whitney test (p-value) & & & \\
PTFE vs. PCD & & 0.002 & \\
PTFE vs. PM & & 0.715 & \\
PTFE vs. PMS & & $<.001$ & \\
PCD vs. PM & & 0.016 \\
PCD vs. PMS & & 0.07 & \\
PM vs. PMS & & 0.001 & \\
\hline
\end{tabular}

$\mathrm{SD}=$ standard deviation; PTFE: polytetrafluoroethylene expanded; PCD: polypropylene encapsulated with polydioxanone and coated with oxidized cellulose; PM: polypropylene mesh; PMS: polypropylene mesh coated with silicone.
Table 4 - Not incorporated area of each type of mesh.

\begin{tabular}{lcccc}
\hline $\begin{array}{l}\text { \% } \\
\text { notincoporatedarea }\end{array}$ & PTFE & PCD & PM & PMS \\
\hline $\mathrm{N}$ & 15 & 15 & 14 & 14 \\
Mena & $42.13 \%$ & $15.47 \%$ & $9.43 \%$ & $14.86 \%$ \\
SD & $10.01 \%$ & $8.26 \%$ & $18.74 \%$ & $18.92 \%$ \\
Median & $44.00 \%$ & $20.00 \%$ & $0.00 \%$ & $8.00 \%$ \\
KW $\chi 2$ & & $\mathbf{2 5 . 6 2 8}$ & \\
$p$ & & $<\mathbf{0 . 0 0 1}$ & \\
Mann-Whitney test $(p$-value) & & & \\
PTFE vs. PCD & & $<0.001$ & \\
PTFE vs. PM & & $<0.001$ & \\
PTFE vs. PMS & & $<0.001$ & \\
PCD vs. PM & & 0.057 & \\
PCD vs. PMS & & 0.331 & \\
PM vs. PMS & & 0.352 & \\
\hline SD & & & \\
\hline
\end{tabular}

$\mathrm{SD}=$ standard deviation; PTFE: polytetrafluoroethylene expanded; PCD: polypropylene encapsulated with polydioxanone and coated with oxidized cellulose; PM: polypropylene mesh; PMS: polypropylene mesh coated with silicone. 
Table 5 - Frequency of hematoma/seroma per type of mesh.

\begin{tabular}{|c|c|c|c|c|c|c|c|c|c|}
\hline \multirow{2}{*}{ Hematoma/seroma } & & \multicolumn{4}{|c|}{ Mesh } & \multirow{2}{*}{ Total } & \multirow{2}{*}{ chi-square } & \multirow{2}{*}{$p$} & \multirow{2}{*}{ Fisher $p$} \\
\hline & & PTFE & PCD & PM & PMS & & & & \\
\hline \multirow{2}{*}{ Yes } & $\mathrm{N}$ & 15 & 10 & 3 & 5 & 33 & \multirow{6}{*}{21.960} & \multirow{6}{*}{$<0.001$} & \multirow{6}{*}{$<0.001$} \\
\hline & $\%$ & $100.00 \%$ & $66.70 \%$ & $21.40 \%$ & $35.70 \%$ & $56.90 \%$ & & & \\
\hline \multirow{2}{*}{ No } & $\mathrm{N}$ & 0 & 5 & 11 & 9 & 25 & & & \\
\hline & $\%$ & $0.00 \%$ & $33.30 \%$ & $78.60 \%$ & $64.30 \%$ & $43.10 \%$ & & & \\
\hline \multirow{2}{*}{ Total } & $\mathbf{N}$ & 15 & 15 & 14 & 14 & 58 & & & \\
\hline & $\%$ & $100.00 \%$ & $100.00 \%$ & $100.00 \%$ & $100.00 \%$ & $100.00 \%$ & & & \\
\hline
\end{tabular}

SD = standard deviation; PTFE: polytetrafluoroethylene expanded; PCD: polypropylene encapsulated with polydioxanone and coated with oxidized cellulose; PM: polypropylene mesh; PMS: polypropylene mesh coated with silicone.

The presence of visceral adhesions to the mesh was similar between groups ( $p=0.029$, Fisher test; $p=8.989$, chi-squared test; data not shown). The presence of adhesion to the omentum was also similar between groups ( $p=0.097$, Fisher test; $p=6.074$, chi-squared test).

The histological analysis revealed that the inflammation scores were significantly different

Table 6 - Inflammation scores per type of mesh.

\begin{tabular}{|c|c|c|c|c|}
\hline Inflammation & PTFE & PCD & PM & PMS \\
\hline N & 15 & 15 & 14 & 14 \\
\hline Mean & 20.93 & 20.2 & 18.64 & 18.21 \\
\hline SD & 0.799 & 0.676 & 1.277 & 1.251 \\
\hline Media & 21 & 20 & 19 & 18 \\
\hline $\mathrm{KW} \times 2$ & \multicolumn{4}{|c|}{35.051} \\
\hline$p$ & \multicolumn{4}{|c|}{$<0.001$} \\
\hline \multicolumn{5}{|c|}{ Mann-Whitney test ( $p$-value) } \\
\hline PTFE vs. PCD & \multicolumn{4}{|c|}{0.009} \\
\hline PTFE vs. PM & \multicolumn{4}{|c|}{$<0.001$} \\
\hline PTFE vs. PMS & \multicolumn{4}{|c|}{$<0.001$} \\
\hline PCD vs. PM & \multicolumn{4}{|c|}{0.001} \\
\hline PCD vs. PMS & \multicolumn{4}{|c|}{$<0.001$} \\
\hline PM vs. PMS & \multicolumn{4}{|c|}{0.361} \\
\hline
\end{tabular}

SD = standard deviation; PTFE: polytetrafluoroethylene expanded; PCD: polypropylene encapsulated with polydioxanone and coated with oxidized cellulose; PM: polypropylene mesh; PMS: polypropylene mesh coated with silicone. between groups, as shown in Table 6 . The investigation of $\mathrm{COX} 2$ by immunohistochemistry in the tissue between the skin and the mesh revealed significantly higher positivity for the ePTFE group (Tables 7 and 8). For the interface between the peritoneum and the mesh, there was also a significant difference between groups (Table 9).

Table 7 - Percentage of skin COX2 positivity in the interface between the skin and the mesh, per type of mesh.

\begin{tabular}{lcccc}
\hline COX2 & PTFE & PCD & PM & PMS \\
\hline N & 12 & 14 & 14 & 13 \\
Mean & 2.17 & 1.21 & 1.14 & 1.31 \\
SD & 0.389 & 0.426 & 0.363 & 0.48 \\
Median & 2 & 1 & 1 & 1 \\
KW x2 & & $\mathbf{2 5 . 5 4 2}$ & \\
$p$ & & $<\mathbf{0 . 0 0 1}$ & \\
Mann-Whitney Test & & & & \\
PTFE vs. PCD & $p$ & & $<0.001$ & \\
PTFE vs. PM & $p$ & & $<0.001$ & \\
PTFE vs. PMS & $p$ & & $<0.001$ & \\
PCD vs. PM & $p$ & & 0.628 & \\
PCD vs. PMS & $p$ & & 0.587 & \\
PM vs. PMS & $p$ & & 0.312 & \\
\hline
\end{tabular}

$\mathrm{SD}$ = standard deviation; PTFE: polytetrafluoroethylene expanded; PCD: polypropylene encapsulated with polydioxanone and coated with oxidized cellulose; PM: polypropylene mesh; PMS: polypropylene mesh coated with silicone. 
Table 8 - COX2 positivity in the interface between the skin and the mesh, per type of mesh.

\begin{tabular}{lcccc} 
COX2 & PTFE & PCD & PM & PMS \\
\hline N & 12 & 14 & 14 & 13 \\
Mean & 2.67 & 1.93 & 1.64 & 1.77 \\
SD & 0.492 & 0.267 & 0.497 & 0.599 \\
Median & 3 & 2 & 2 & 2 \\
KW $x 2$ & & $\mathbf{2 1 . 3 5 7}$ & \\
P & & $<\mathbf{0 . 0 1}$ & \\
Mann-Whitney Test & & & & \\
PTFE vs. PCD & $p$ & & $<.001$ & \\
PTFE vs. PM & $p$ & & $<.001$ & \\
PTFE vs. PMS & $p$ & & 0.001 & \\
PCD vs. PM & $p$ & & 0.07 & \\
PCD vs. PMS & $p$ & & 0.315 & \\
PM vs. PMS & $p$ & & 0.605 & \\
\hline
\end{tabular}

$\mathrm{SD}=$ standard deviation; PTFE: polytetrafluoroethylene expanded; PCD: polypropylene encapsulated with polydioxanone and coated with oxidized cellulose; PM: polypropylene mesh; PMS: polypropylene mesh coated with silicone.

\section{- Discussion}

Although the correction of abdominal wall hernias with the mesh is one of the most frequent interventions in the daily life of the general surgeon, there are still many doubts about the host response to various types of mesh used in this procedure. Intraperitoneal mesh, sometimes with direct contact with abdominal organs, has been increasingly used ${ }^{21}$ and there are more than 600 products available ${ }^{22}$, most still pending clinical trials before use in humans. A gap in the literature, that this study tried to fill, was the study of inflammatory reactions, scarring and postoperative complications with different types of products, as adherences, intestinal fistulae and infection are common complications ${ }^{23,24}$. The idea of coating mesh with physical or chemical barriers to adherences was promising, but these substances could potentially impair incorporation and increase the risk of infection. In this study, the polypropylene mesh coated with silicone (PMS) had the best incorporation and the lowest area of adherences.

The higher rates of visceral adherences with the polypropylene mesh (PM) in this study was in accordance with some other studies ${ }^{21,25}$. Other authors ${ }^{26}$ found no significant differences in visceral adherences, suggesting that absorbable compounds that cover the mesh, with late degradation, could be the explanation. It was proposed that mesh covered with Goretex or expanded polytetrafluoroethylene (ePTFE) would create a "neoperitoneum" 27 , preventing
Table 9 - COX2 positivity in the interface between the peritoneum and the mesh, per type of mesh.

\begin{tabular}{lcccc} 
COX2 & PTFE & PCD & PM & PMS \\
\hline N & 12 & 14 & 14 & 13 \\
Mean & 1.75 & 1.36 & 1.14 & 1 \\
SD & 0.754 & 0.497 & 0.363 & 0 \\
Median & 2 & 1 & 1 & 1 \\
KW x2 & & $\mathbf{1 3 . 0 5 9}$ & \\
$p$ & & $\mathbf{0 . 0 0 5}$ & \\
Mann-Whitney Test (p-value) & & \\
PTFE vs. PCD & & 0.164 & \\
PTFE vs. PM & & 0.017 & \\
PTFE vs. PMS & & 0.002 & \\
PCD vs. PM & & 0.199 \\
PCD vs. PMS & & 0.019 \\
PM vs. PMS & & 0.165 \\
\hline
\end{tabular}

SD = standard deviation; PTFE: polytetrafluoroethylene expanded; PCD: polypropylene encapsulated with polydioxanone and coated with oxidized cellulose; PM: polypropylene mesh; PMS: polypropylene mesh coated with silicone.

the adherence to abdominal viscera ${ }^{25,27}$. However, the absorbable material could also increase the inflammatory process ${ }^{26,28}$, as in fact observed in our study, in which the ePTFE group presented higher incidence of collections along its surface (100\%), while polypropylene mesh (PM) had the lowest (21\%; $\mathrm{p}<0.001)$. Contradictory results in other studies ${ }^{28,29}$ may be due to differences in the experimental animals, mesh porosity and experimental or surgical methodology.

There are studies in the literature also in Wistar rats evaluating the intraperitoneal adherences that are seen with mesh covered with silicone. Takácset al. ${ }^{30}$ compared two different silicone mesh products and observed lower adherence with the laminar surgical silicon mesh. Baracs et al. ${ }^{31}$ obtained results that were similar to ours. In their study, they compared a silicone-covered mesh with polypropylene meshes and observed that the silicone-covered product significantly decreased the formations of adhesion. However, our study was more comprehensive: besides comparing the silicone with the polypropylene mesh, we also compared the abdominal adhesions with the ePTFE and the PCD mesh, which are products used in the intraperitoneal position. In this study, the PMS group presented the lowest adhesion area (63.1\%) and grade 1 adhesion (22.4\%) among all the evaluated products. The PCD group showed an intermediate 
area of adhesion and a looser adhesion (grade 2), and the ePTFE and PM groups presented almost the total area of adherence (99.8\% and $97.7 \%$, respectively). However, grade 2 adhesions predominated in the ePTFE group and grade 4 in the PM (86.7\% and 64.3\%). As the mesh in the PCD group contained absorbable material, it resulted in a greater inflammatory process and adhesions than in the ePTFE and PMS groups. We believe that the PMS group was the mesh that presented less intraperitoneal adhesion and between the viscera and the mesh due to the fact that it does not contain absorbable material and presents a longlasting silicone barrier.

In our study, we observed that the group ePTFE had the higher percentage of area without incorporation (42\%; $p<0.001$ ), while the PCD, PM e PMS groups had no significant difference between them (15.4\%; 9.4\%; e $14.8 \%$ respectively). In the study by Schreinemacheret al. ${ }^{32}$, three types of meshes were compared: polypropylene, polypropylene coated with carboxymethyl cellulose and polypropylene coated with ePTFE, without significant differences between them. In the experiment by Raptiset $a .^{24}$, the ePTFE mesh was not well incorporated, since it became encapsulated when fixed intraperitoneally. As the incorporation of the meshes is related to fibroblasts and collagen infiltration in the material and it is directly associated with the inflammatory process caused by the product, we believe that, in the case of the ePTFE mesh, the presence of micropores hinders incorporation, since the mesh prevents the free passage and the infiltration of these components into its structure. The PMS group presented better incorporation than the ePTFE Group and no difference was observed in relation to the groups containing polypropylene. Possibly these findings occurred because the parietal face of the mesh in the PMS group has polypropylene in one of its surfaces, similar to the PCD and PM groups and different from the ePTFE mesh which, although presenting a texture difference, is made of the same material on both surfaces.

No other investigators studied COX2 in hernia repair mesh products, and we have shown a higher inflammation score, evaluated by the COX2 analysis, with the ePTFE group in this study $(p<0.001)$ - while in the studies by Raptiset al. ${ }^{24}$ and Matthews et al. ${ }^{25}$, the ePTFE had the lowest inflammatory reaction. COX2 is a proinflammatory substance that is the precursor to the arachidonic acid pathway, also involved with angiogenesis in inflammatory and neoplastic processes. These stable molecules are classically considered markers of inflammatory response ${ }^{20}$. There are no other similar investigations of COX 2 by immunohistochemistry in mesh studies. In our group, Pereira-Lucena et al. ${ }^{19}$ investigated COX2 in a mesh fixed extraperitoneally, observing a higher concentration and percentage of this marker in the polypropylene mesh. In our results, the ePTFE group presented the highest inflammatory response, both by the scoring histological evaluation and in the COX2 immunohistochemistry evaluation, which confirms the greater inflammation caused by the mesh. There is still doubt as to whether this inflammatory response occurred due to the presence of the collection, or as a response of the mesh itself. This should be further investigated regarding a possible multifactorial cause for the inflammation: the presence of seroma (as mentioned by Raptis et $a .^{24}$ ), the size of pores, the mesh density and different materials used in coating. The comparisons should be made in studies in which there is an experimental suture fixing mesh to the abdominal wall, and not only intraperitoneal insertion of mesh products with no replacement of parietal tissues.

Deerenberg et al. $^{33}$ used the same mesh and same surgical procedure used in our study, but in a contaminated environment. The ePTFE had the worst result in that scenario, both in incorporation and infection rates, because the micropores probably facilitated the infiltration and proliferation of bacteria and impaired the action of defense cells. Besides, its visceral hydrophobic face would decrease tissue cell adhesion and allow the free passage of bacteria to the implant surface.

\section{- Conclusions}

Among all types of mesh studied, the polypropylene mesh (PM) had higher density of adherences, larger area of adherences, adherences to organs and percentage of incorporation. The polytetrafluoroethylene expanded mesh (ePTFE) had the higher area of adherences and lower incorporation. The polypropylene mesh coated with silicone (PMS) performed best in inflammation score, had a higher incorporation and lower area of adherences, and it was considered the best type of mesh regarding inflammation, adherences and incorporation. However, this is an experimental study with rats, with short follow-up, and there are no other studies that could be compared to ours in the literature yet.

\section{- References}

1. Deerenberg EB, Timmermans L, Hogerzeil DP, Slieker JC, Eilers PH, Jeekel J, Lange JF. A systematic review of the surgical treatment of large incisional hernia. Hernia. 2015 Feb;19(1):89-101. doi: 10.1007/s10029-014-1321-x. 
2. Ohana G, Bramnik Z, Miller A, Seror D, Ariche A, Bachar Gil N, Belavsky R, Dreznik Z. Treatment of large incisional abdominal wall hernias, using a modified preperitoneal prosthetic mesh repair. Hernia. 2006 Jun;10(3):232-5.doi: 10.1007/s10029-006-0070-x.

3. Anthony $T$, Bergen PC, Kim LT, Henderson M, Fahey T, Rege RV, Turnage RH. Factors affecting recurrence following incisional herniorrhaphy. World J Surg. 2000 Jan;24(1):95100; discussion 101. PMID: 10594211.

4. Burger JW, Luijendijk RW, Hop WC, Halm JA, Verdaasdonk $E G$, Jeekel J. Long-term follow-up of a randomized controlled trial of suture versus mesh repair of incisional hernia. Ann Surg. 2004 Oct;240(4):578-83; discussion 5835. PMID: 15383785.

5. Cobb WS, Harris JB, Lokey JS, McGill ES, Klove KL. Incisional herniorrhaphy with intraperitoneal composite mesh: a report of 95 cases. Am Surg. 2003 Sep;69(9):784-7. PMID: 14509327.

6. Cobb WS, Kercher KW, Matthews BD, Burns JM, Tinkham $\mathrm{NH}$, Sing RF, Heniford BT. Laparoscopic ventral hernia repair: a single center experience. Hernia. 2006 Jun;10(3):236-42. doi: 10.1007/s10029-006-0072-8.

7. Arnaud JP, Patsopoulos J, Adloff M. Treatment of voluminous event rations of the abdominal wall: mersilene mesh in intraperitoneal position associated with an aponeurotic graft. Apropos of 96 cases. Ann Chir. 1983 Jun;37(5):337-40. PMID: 6225365.

8. de VriesReilingh TS, van Geldere D, Langenhorst B, de Jong D, van der Wilt GJ, van Goor H, Bleichrodt RP. Repair of large midline incisional hernias with polypropylene mesh: comparison of three operative techniques. Hernia. 2004 Feb;8(1):56-9. doi: 10.1007/s10029-003-0170-9.

9. Farmer L, Ayoub M, Warejcka D, Southerland S, Freeman A, Solis M. Adhesion formation after intraperitoneal and extraperitoneal implantation of polypropylene mesh. Am Surg. 1998 Feb;64(2):144-6. PMID: 9486886.

10. Losanoff JE, Richman BW, Jones JW. Entero-colocutaneous fistula: a late consequence of polypropylene mesh abdominal wall repair: case report and review of the literature. Hernia. 2002 Sep;6(3):144-7. doi: 10.1007/ s10029-002-0067-z.

11. Ott V, Groebli Y, Schneider R. Late intestinal fistula formation after incisional hernia using intraperitoneal mesh. Hernia. 2005 Mar;9(1):103-4. doi: 10.1007/s10029004-0271-0.

12. Vrijland WW, Jeekel J, Steyerberg EW, Den Hoed PT, Bonjer HJ. Intraperitoneal polypropylene mesh repair of incisional hernia is not associated with enterocutaneous fistula. Br J Surg. 2000 Mar;87(3):348-52. doi: 10.1046/j.13652168.2000.01364.x.

13. Alponat A, Lakshminarasappa SR, Yavuz N, Goh PM. Prevention of adhesions by Seprafilm, an absorbable adhesion barrier: an incisional hernia model in rats. Am Surg. 1997 Sep;63(9):818-9. PMID: 9290528.

14. Hooker GD, Taylor BM, Driman DK. Prevention of adhesion formation with use of sodium hyaluronate-based bioresorbable membrane in a rat model of ventral hernia repair with polypropylene mesh--a randomized, controlled study. Surgery. 1999 Feb;125(2):211-6. PMID: 10026756.
15. Zühlke HV, Lorenz EM, Straub EM, Savvas V. Pathophysiology and classification of adhesions. Langenbecks Arch Chir Suppl II Verh Dtsch Ges Chir. 1990:1009-16. PMID: 1983476.

16. Rose J, Jayaraman S, Colquhoun P, Taylor B. Minimal abdominal adhesions after Sepramesh repair of a parastomal hernia. Can J Surg. 2009 Oct;52(5):E211-2. PMID: 19865564.

17. Arung $W$, Drion $P$, Cheramy JP, Honoré $P$, Meurisse $M$, Defraigne JO, Detry O. Intraperitoneal adhesions after open or laparoscopic abdominal procedure: an experimental study in the rat. J Laparoendosc Adv Surg Tech A. 2012 Sep;22(7):651-7. doi: 10.1089/lap.2012.0102.

18. Harrell AG, Novitsky YW, Cristiano JA, Gersin KS, Norton HJ, Kercher KW, Heniford BT. Prospective histologic evaluation of intra-abdominal prosthetics four months after implantation in a rabbit model. Surg Endosc. 2007 Jul;21(7):1170-4. doi: 10.1007/s00464-006-9147-y.

19. Pereira-Lucena CG, Artigiani-Neto R, Lopes-Filho GJ, Frazao CV, Goldenberg A, Matos D, Linhares MM. Experimental study comparing meshes made of polypropylene, polypropylene + polyglactin and polypropylene + titanium: inflammatory cytokines, histological changes and morphometric analysis of collagen. Hernia. 2010 Jun;14(3):299-304. doi: 10.1007/s10029-009-0621-z.

20. Kim HS, Lee HS, Kim WH. Clinical significance of protein expression of cyclooxygenase-2 and somatostatin receptors in gastroenteropancreatic neuroendocrine tumors. Cancer Res Treat. 2011 Sep;43(3):181-8. doi: 10.4143/crt.2011.43.3.181.

21. Jenkins ED, Yom V, Melman L, Brunt LM, Eagon JC, Frisella MM, Matthews BD. Prospective evaluation of adhesion characteristics to intraperitoneal mesh and adhesiolysisrelated complications during laparoscopic re-exploration after prior ventral hernia repair. Surg Endosc. 2010 Dec;24(12):3002-7. doi: 10.1007/s00464-010-1076-0.

22. Klinge $U$, Klosterhalfen $B$. Modified classification of surgical meshes for hernia repair based on the analyses of 1,000 explanted meshes. Hernia. 2012 Jun;16(3):251-8. doi: 10.1007/s10029-012-0913-6.

23. Brandi $C D$, Roche $S$, Bertone $S$, Fratantoni ME. No enterocutaneous fistula development in a cohort of 695 patients after incisional hernia repair using intraperitoneal uncoated polyproylene mesh. Hernia. 2017 Feb;21(1):101106. doi: 10.1007/s10029-016-1530-6.

24. Raptis DA, Vichova B, Breza J, Skipworth J, Barker S.A comparison of woven versus nonwoven polypropylene (PP) and expanded versus condensed polytetrafluoroethylene (PTFE) on their intraperitoneal incorporation and adhesion formation. J Surg Res. 2011 Jul;169(1):1-6. doi: 10.1016/j. jss.2009.12.014.

25. Matthews BD, Mostafa G, Carbonell AM, Joels CS, Kercher KW, Austin C, Norton HJ, Heniford BT. Evaluation of adhesion formation and host tissue response to intraabdominal polytetrafluoroethylene mesh and composite prosthetic mesh. J Surg Res. 2005 Feb;123(2):227-34. doi: 10.1016/j.jss.2004.08.012.

26. Schreinemacher MH, Emans PJ, Gijbels MJ, Greve JW, Beets GL, Bouvy ND. Degradation of mesh coatings and intraperitoneal adhesion formation in an experimental model. Br J Surg. 2009 Mar;96(3):305-13. doi: 10.1002/bjs.6446. 
27. Zieren J, Paul M, Osei-Agyemang T, Maecker F, Müller JM Polyurethane-covered dacron mesh versus polytetrafluoroethylene Dual Mesh for intraperitoneal hernia repair in rats. Surg Today. 2002;32(10):884-6. doi: $10.1007 / \mathrm{s} 005950200172$.

28. Reynvoet E, Chiers K, Van Overbeke I, Troisi R, Berrevoet F. Intraperitoneal mesh devices for small midline hernias: mesh behavior in a porcine model. Hernia. 2015 Dec;19(6):955-63. doi: 10.1007/s10029-015-1368-3.

29. Konarzewski NS, Bigolin A, Montes J, Lambert B, Kist C, Grossi JV, Cavazzola LT. Evaluation of intraperitoneal adhesions associated with the double layer Mesh PTFEe/Polypropylene in the ventral hernia repair - an experimental study in rats. Braz J Videoendoscopic. 2009;2(1):2-10.

30. Takács I, Horváth $S$, Molnár Á, Gáspár S, Hajós R, Meczker Á, Kóbor P, Lantos J, Jávor S, Balatonyi B, Szekeres G, Röth
E, Wéber G. Comparative immunohistochemical study of tissue integration of macroporous and laminar surgical meshes. Histol Histopathol. 2011 Jul;26(7):821-30. doi: 10.14670/HH-26.821.

31. Baracs J, Takács I, Shahram GS. Biological behavior of polypropylene meshes suitable for intra-abdominal implantation in animal model. MagySeb. 2003 Oct;56(5):171-6.PMID: 15022620.

32. Schreinemacher $\mathrm{MH}$, van Barneveld KW, Dikmans RE, Gijbels MJ, Greve JW, Bouvy ND. Coated meshes for hernia repair provide comparable intraperitoneal adhesion prevention. Surg Endosc. 2013 Nov;27(11):4202-9. doi: 10.1007/s00464-013-3021-5.

33. Deerenberg EB, Mulder IM, Grotenhuis N, Ditzel M, Jeekel J, Lange JF. Experimental study on synthetic and biological mesh implantation in a contaminated environment. $\mathrm{Br} \mathrm{J}$ Surg. 2012 Dec;99(12):1734-41. doi: 10.1002/bjs.8954.

\section{Correspondence:}

Rogério Aoki Fuziy

Rua Napoleão de Barros, 715/20 andar

04024-002 São Paulo - SP Brasil

Tel.: (55 11)98685-4449

rfuziy@gmail.com

Received: Mar 16, 2019

Review: May 14, 2019

Accepted: June 13, 2019
Conflict of interest: none

Financial source: none

This is an Open Access article distributed under the terms of the Creative Commons Attribution License, which permits unrestricted use, distribution, and reproduction in any medium, provided the original work is properly cited.

1 Research performed at Division of Surgical Gastroenterology, Department of Surgery, Universidade Federal de São Paulo (UNIFESP), Brazil. Part of Master degree thesis, Postgraduate Program in Interdisciplinary Surgical Sciences, UNIFESP. Tutor: Gaspar Jesus Lopes Filho. 